\title{
PRESERVATION OF PSEUDO-METRIZABILITY BY QUOTIENT MAPS ${ }^{1}$
}

\author{
C. J. HIMMELBERG ${ }^{2}$
}

In this paper we obtain necessary and sufficient conditions for a quotient map to preserve pseudo-metrizability. All notation and terminology not explicitly developed here are as in [1]. We assume throughout that $f$ is a given function from a set $X$ on to a set $Y$. Moreover, we will need the following theorem, whose proof is the same as the proof of Theorem 2 in [1].

THEOREM 1. Let $\boldsymbol{u}$ be a uniformity for $X$ such that $\boldsymbol{f}(\mathcal{u})$ is a uniformity for $Y$, and give $X$ the topology of $\mathcal{u}$. Then the quotient topology on $Y$ relative to $f$ is the topology of the quotient uniformity $f(\mathcal{U})$ if, for each member $G$ of some base of the quotient topology on $Y$ and for each $y \in G$, there exists $U \in \mathcal{U}$ such that $U\left[f^{-1}[y]\right] \subset f^{-1}[G]$.

Recall that a uniformity $\mathcal{u}$ for $X$ can be defined by a single pseudometric if and only if $u$ has a countable base. Moreover, $f(\mathcal{u})$ has a countable base whenever $\mathfrak{u}$ does. Hence we obtain immediately the following.

Theorem 2. Suppose that $X$ is a pseudo-metrizable space and that there is a pseudo-metric and corresponding uniformity $u$ for $X$ which yields the given topology on $X$ and is such that $\boldsymbol{f}(\mathcal{u})$ is a uniformity. Suppose further that, for each member $G$ of some base of the quotient topology on $Y$ and for each $y \in G$, there exists $U \in \mathcal{U}$ such that $U\left[f^{-1}[y]\right] \subset f^{-1}[G]$. (This last condition is satisfied if, for example, $f^{-1}[y]$ is compact for each $y \in G$.) Then the quotient topology on $Y$ relative to $f$ is pseudometrizable.

Our intention now is to obtain a theorem on preservation of pseudo-metrizability which does not require that $f[u]$ be a uniformity. We will need some notational conventions. If $(W, d)$ is a pseudo-metric space, $d$ is allowed to take the value $\infty$, since any such pseudo-metric can be replaced by a bounded one without changing the underlying uniform structure. If $g$ is a function from $W$ to $Y$, $y \in Y$, and $\epsilon>0$, then $N_{\epsilon}\left[g^{-1}[y]\right]=\left\{x \in W \mid d\left(x, g^{-1}[y]\right)<\epsilon\right\}$. If, in

Received by the editors October 10, 1964.

1 This research was supported by the National Science Foundation (GP-2557).

2 The author is indebted to the referee for several useful remarks, and in particular for proving the "only if" part of Theorem 3. 
addition, $Y$ has a topology, we say that $W, d, Y, g$ satisfy condition (C) if and only if the following condition is satisfied.

(C) For each member $G$ of some base of $Y$ there exists a family $\{\epsilon(y) \mid y \in G\}$ of positive real numbers such that

$$
N_{\epsilon(y)}\left[g^{-1}[y]\right] \subset g^{-1}[G], \quad \text { if } y \in G,
$$

and

$$
d\left(g^{-1}[y], g^{-1}[z]\right) \geqq \epsilon(y)-\epsilon(z), \quad \text { if } y, z \in G .
$$

Theorem 3. Let $X$ be a pseudo-metrizable space. Then the quotient topology on $Y$ relative to $f$ is pseudo-metrizable if and only if the topology on $X$ can be defined by a pseudo-metric d such that $X, d, Y, f$ satisfy $(\mathrm{C})$.

Proof. Suppose that the quotient topology on $Y$ is pseudo-metrizable, say by a bounded pseudo-metric $d_{Y}$, and let $d_{X}$ be any pseudometric consistent with the topology of $X$. Define $d(x, y)=d_{X}(x, y)$ $+d_{Y}(f(x), f(y))$ for $x, y \in X$. Then $d$ is a pseudo-metric on $X$, giving the same topology as $d_{X}$. It is now easy to see that $X, d, Y, f$ satisfy (C). For if $G$ is any proper open subset of $Y$, let $\epsilon(y)=d_{Y}(y, Y-G)$ for each $y \in G$, and then verify (i) and (ii) of (C).

On the other hand, suppose that $Y$ has the quotient topology and that $d$ is a pseudo-metric for $X$ such that $X, d, Y, f$ satisfy (C). In the sequence of propositions to follow this proof we will construct a pseudo-metrizable space $X_{\infty}$ containing $X$, a pseudo-metric $d_{\infty}$ defining the topology of $X_{\infty}$, and an extension of $f$ to a quotient map $f_{\infty}: X_{\infty} \rightarrow Y$ such that: for each $G$ in the base described in (C) and each $y \in G$ there exists $\epsilon>0$ for which $N_{\epsilon}\left[f_{\infty}^{-1}[y]\right] \subset f_{\infty}^{-1}[G]$; and $\boldsymbol{f}_{\infty}\left[\mathfrak{u}_{\infty}\right]$ is a uniformity, where $\boldsymbol{f}_{\infty}$ is defined by $\boldsymbol{f}_{\infty}(x, y)=\left(f_{\infty}(x), f_{\infty}(y)\right)$ for $x$, $y \in X_{\infty}$ and $\mathcal{u}_{\infty}$ is the uniformity defined by $d_{\infty}$. It then follows from Theorem 2 that the quotient topology on $Y$ is pseudo-metrizable.

It remains to construct $X_{\infty}, d_{\infty}, f_{\infty}$ given $X, d, f$.

For each ordered pair $(u, v)$ of points of $X$ such that $f(u)=f(v)$, let $\phi$ be an isometry from $X$ on to a space $\phi[X]$ whose intersection with $X$ is $v$ and which is such that $\phi(u)=v$. Let $\Phi$ be the set of isometries chosen in this way. For each $X \cup_{\phi}[X]$ define a pseudo-metric $d_{\phi}$ by

$$
\begin{aligned}
d_{\phi}(x, y) & =d(x, y), \quad \text { if } x, y \in X, \\
& =d\left(\phi^{-1}[x], \phi^{-1}[y]\right), \quad \text { if } x, y \in \phi[X], \\
& =d(x, v)+d\left(u, \phi^{-1}[y]\right), \quad \text { if } x \in X, y \in \phi[X], \\
& =d\left(\phi^{-1}[x], u\right)+d(v, y), \quad \text { if } x \in \phi[X], y \in X,
\end{aligned}
$$

and extend $f$ to the function $f_{\phi}: X \cup \phi[X] \rightarrow Y$ defined by 


$$
\begin{aligned}
f_{\phi}(x) & =f(x), \quad \text { if } x \in X, \\
& =f \circ \phi^{-1}[x], \quad \text { if } x \in \phi[X] .
\end{aligned}
$$

Next regard any two of the spaces $X \cup \phi[X], X \cup \psi[X]$, with $\phi, \psi$ distinct members of $\Phi$, as disjoint, and let

$$
X_{1}=\cup\{X \cup \phi[X] \mid \phi \in \Phi\} .
$$

Define a pseudo-metric $d_{1}$ for $X_{1}$ by

$$
\begin{aligned}
d_{1}(x, y) & =d_{\phi}(x, y), \quad \text { if } x, y \in X \cup \phi[X], \phi \in \Phi, \\
& =\infty, \quad \text { otherwise, }
\end{aligned}
$$

and extend all $f_{\phi}$ 's simultaneously to the function

$$
f_{1}=\bigcup\left\{f_{\phi} \mid \phi \in \Phi\right\}: X_{1} \rightarrow Y .
$$

We regard $X_{1}$ as disjoint from $X$, although we keep in mind that $X_{1}$ contains $\Phi$ copies of $X$, on each of which $f_{1}, d_{1}$ behave exactly like $f, d$, respectively.

Obtain a sequence of mutually disjoint pseudo-metric spaces $(X, d)=\left(X_{0}, d_{0}\right),\left(X_{1}, d_{1}\right), \cdots,\left(X_{n}, d_{n}\right), \cdots$ and functions $f_{0}$ $=f: X \rightarrow Y, f_{1}: X_{1} \rightarrow Y, \cdots, f_{n}: X_{n} \rightarrow Y, \cdots$, by constructing $\Phi_{n}$, $X_{n+1}, d_{n+1}, f_{n+1}$ from $X_{n}, d_{n}, f_{n}$ in the same way that $\Phi, X_{1}, d_{1}, f_{1}$ were constructed from $X, d, f$. If $m<n$, then $X_{n}$ contains $\Phi_{m} \times \cdots$ $\times \Phi_{n-1}$ copies of $X_{m}$, on each of which $f_{n}, d_{n}$ behave exactly like $f_{m}, d_{m}$, respectively. Now define

$$
\begin{aligned}
X_{\infty}=\bigcup\left\{X_{n} \mid n\right. & =0,1, \cdots\}, \quad f_{\infty}=\bigcup\left\{f_{n} \mid n=0,1, \cdots\right\}, \\
d_{\infty}(x, y) & =d_{n}(x, y), \quad \text { if } x, y \in X_{n}, \\
& =\infty, \quad \text { if } x \in X_{m}, y \in X_{n}, m \neq n .
\end{aligned}
$$

In the propositions to follow we need the notation defined below.

$$
\begin{array}{ll}
R_{n}[x]=\left\{z \in X_{n} \mid f_{n}(z)=f_{\infty}(x)\right\}, & \text { if } x \in X_{\infty}, \\
R_{\infty}[x]=\left\{z \in X_{\infty} \mid f_{\infty}(z)=f_{\infty}(x)\right\}, & \text { if } x \in X_{\infty} .
\end{array}
$$

Observe that

$$
\begin{aligned}
& R_{0}[x]=R[x], \quad \text { if } x \in X, \\
& R_{\infty}[x]=\bigcup\left\{R_{n}[x] \mid n=0,1, \cdots\right\}, \quad \text { if } x \in X_{\infty} .
\end{aligned}
$$

Proposition 1. Suppose $\epsilon>0$ and that $x, y, z \in X_{x}$ are such that $d_{\infty}\left(x, R_{n}[z]\right)<\epsilon / 2$ and $d_{\infty}\left(y, R_{n}[z]\right)<\epsilon / 2$. Then $d_{\infty}\left(R_{n+1}[x], R_{n+1}[y]\right)$ $<\epsilon$.

Proof. Clearly, $x, y \in X_{n}$. Let $u, v \in R_{n}[z]$ be such that $d_{n}(x, u)$ 
$<\epsilon / 2, d_{n}(y, v)<\epsilon / 2$, and let $\phi \in \Phi_{n}$ be such that $\phi(u)=v$. Then $\phi(x) \in R_{n+1}[x]$, and we let $y$ represent both itself as a point of $X_{n}$ and the point of $X_{n} \cup_{\phi}\left[X_{n}\right]$ which corresponds to it under the identity injection. It follows that

$$
\begin{aligned}
d_{\infty}\left(R_{n+1}[x], R_{n+1}[y]\right) & =d_{n+1}\left(R_{n+1}[\phi(x)], R_{n+1}[y]\right) \leqq d_{n+1}(\phi(x), y) \\
& =d_{n}(y, v)+d_{n}(x, u)<\epsilon .
\end{aligned}
$$

Proposition 2. Suppose $\epsilon>0$ and that $x, y, z \in X_{\infty}$ are such that $d_{\infty}\left(x, R_{\infty}[z]\right)<\epsilon / 2, d_{\infty}\left(y, R_{\infty}[z]\right)<\epsilon / 2$. Then $d_{\infty}\left(R_{\infty}[x], R_{\infty}[y]\right)<\epsilon$.

Proof. There exist $m, n$ such that $x \in X_{m}, y \in X_{n}$. Hence $d_{\infty}\left(x, R_{m}[z]\right)$ $<\epsilon / 2$ and $d_{\infty}\left(y, R_{n}[z]\right)<\epsilon / 2$. Suppose $m \leqq n$. By an easy inductive argument it follows that there exists $w \in R_{n}[x] \subset R_{\infty}[x]$ such that $d_{\infty}\left(w, R_{n}[z]\right)<\epsilon / 2$. So, by Proposition 1 , we have

$$
d_{\infty}\left(R_{\infty}[x], R_{\infty}[y]\right) \leqq d_{\infty}\left(R_{n+1}[w], R_{n+1}[y]\right)<\epsilon .
$$

Proposition 3. If $\mathfrak{u}_{\infty}$ is the uniformity for $X_{\infty}$ defined by $d_{\infty}$, then $\boldsymbol{f}_{\infty}\left[\mathfrak{u}_{\infty}\right]$ is a uniformity.

Proof. Apply Proposition 2 to (d) of Theorem 1 in [1].

Proposition 4. Suppose $X, d, Y, f$ satisfy condition (C). If $n=0,1, \cdots$, then $X_{n}, d_{n}, Y, f_{n}$ also satisfy $(\mathrm{C})$, with, for each basic open subset $G$ of $Y$, the same choice of $\{\epsilon(y) \mid y \in G\}$ that is used for $X$, $d, Y, f$.

Proof. By induction. The proposition is trivially true for $n=0$. The argument establishing the induction step is the same, except for notation, as the proof that $X_{1}, d_{1}, Y, f_{1}$ satisfy (C). This being the case, we give here only the latter.

Observe that, since

$$
N_{\epsilon}\left[f_{1}^{-1}[y]\right]=\cup\left\{N_{\epsilon}\left[f_{\phi}^{-1}[y]\right] \mid \phi \in \Phi\right\}, \quad \text { if } \epsilon>0 \text { and } y \in Y,
$$

and

$$
\left.d_{1}\left(f_{1}^{-1}[y], f_{1}^{-1}[z]\right)=\inf \left\{d_{\phi}\left({f_{\phi}^{-1}}^{-1}\right],{\overrightarrow{f_{\phi}}}^{-1}[z]\right) \mid \phi \in \Phi\right\}, \quad \text { if } y, z \in Y,
$$

it is clearly sufficient to show that $X \cup \phi[X], d_{\phi}, Y, f_{\phi}$ satisfy (C) with, for each basic open subset $G$ of $Y$, the same choice of $\{\epsilon(y) \mid y \in G\}$ that works for $X, d, Y, f$.

So let $u, v \in X$ be such that $f(u)=f(v)=w$, and let $\phi$ be the isometry of $X$ such that $\phi(u)=v$. Let $G$ be a basic open subset of $Y$, and let $\{\epsilon(y) \mid y \in G\}$ be chosen as in (C) applied to $X, d, Y, f$. Then it remains to prove 


$$
N_{\epsilon(y)}\left[f_{\phi}^{-1}[y]\right] \subset f_{\phi}^{-1}[G], \quad \text { if } y \in G,
$$

and

$$
d_{\phi}\left(f_{\phi}^{-1}[y], f_{\phi}^{-1}[z]\right) \geqq \epsilon(y)-\epsilon(z), \quad \text { if } y, z \in G .
$$

To prove (i), suppose $d_{\phi}\left(x, f_{\phi}^{-1}[y]\right)<\epsilon(y), y \in G$. Since $f_{\phi}^{-1}[y]$ $=f^{-1}[y] \cup \phi \circ f^{-1}[y]$, we consider four cases:

Case 1. $x \in X, d\left(x, f^{-1}[y]\right)<\epsilon(y)$,

Case 2. $x \in X, d_{\phi}\left(x, \phi \circ f^{-1}[y]\right)<\epsilon(y)$,

Case 3. $x \in \phi[X], d_{\phi}\left(x, \phi \circ f^{-1}[y]\right)<\epsilon(y)$,

Case 4. $x \in \phi[X], d_{\phi}\left(x, f^{-1}[y]\right)<\epsilon(y)$.

Since $f_{\phi}^{-1}[G]=f^{-1}[G] \cup \phi \circ f^{-1}[G]$, it follows easily from Case 1 or Case 3 that $x \in f_{\phi}^{-1}[G]$. Cases 2 and 4 are similar, and we give the details only for Case 2. We have in this case

$$
d_{\phi}\left(x, \phi \circ f^{-1}[y]\right)=d(x, v)+d\left(u, f^{-1}[y]\right)<\epsilon(y) .
$$

Hence $u \in f^{-1}[G]$ and $w=f(u) \in G$. Moreover,

$$
d(x, v)<\epsilon(y)-d\left(u, f^{-1}[y]\right) \leqq \epsilon(y)-d\left(f^{-1}[w], f^{-1}[y]\right) \leqq \epsilon(w),
$$

so that $x \in N_{\mathrm{e}(w)}\left[f^{-1}[w]\right] \subset f^{-1}[G] \subset f_{\phi}^{-1}[G]$.

To prove (ii), let $y, z \in G$, and observe that $d_{\phi}\left(f_{\phi}^{-1}[y], f_{\phi}^{-1}[z]\right)$ is the minimum of the four quantities $d\left(f^{-1}[y], f^{-1}[z]\right), d_{\phi}\left(\phi \circ f^{-1}[y], \phi \circ f^{-1}[z]\right)$, $d_{\phi}\left(f^{-1}[y], \phi \circ f^{-1}[z]\right), d_{\phi}\left(\phi \circ f^{-1}[y], f^{-1}[z]\right)$. We consider only the case in which

$$
d_{\phi}\left(f_{\phi}^{-1}[y], f_{\phi}^{-1}[z]\right)=d_{\phi}\left(f^{-1}[y], \phi \circ f^{-1}[z]\right),
$$

the others being either similar or trivial. If $w \in G$, we have

$$
\begin{aligned}
d_{\phi}\left(f^{-1}[y], \phi \circ f^{-1}[z]\right) & =d\left(f^{-1}[y], v\right)+d\left(u, f^{-1}[z]\right) \\
& \geqq d\left(f^{-1}[y], f^{-1}[w]\right)+d\left(f^{-1}[w], f^{-1}[z]\right) \\
& \geqq \epsilon(y)-\epsilon(z) .
\end{aligned}
$$

If $w \in G$, there is no point of $f^{-1}[w]$ in either $V_{\epsilon(y)}\left[f^{-1}[y]\right]$ or in $V_{\epsilon(z)}\left[f^{-1}[z]\right]$. Hence,

$$
\begin{aligned}
d_{\phi}\left(f^{-1}[y], \phi \circ f^{-1}[z]\right) & \geqq d\left(f^{-1}[y], f^{-1}[w]\right)+d\left(f^{-1}[w], f^{-1}[z]\right) \\
& \geqq \epsilon(y)+\epsilon(z) \geqq \epsilon(y)-\epsilon(z) .
\end{aligned}
$$

Proposition 5. If $X, d, Y, f$ satisfy (C), then so also do $X_{\infty}, d_{\alpha}$, $Y_{\infty}, f_{\infty}$, with, for each basic open subset $G$ of $Y$, the same choice of $\{\epsilon(y) \mid y \in G\}$ that is used for $X, d, Y . f$.

Proof. This proposition follows from the previous one. For let $G$ 
be a basic open subset of $Y$ and let $\{\epsilon(y) \mid y \in G\}$ be as in (C) applied to $X, d, Y, f$. Then

$$
N_{\epsilon(y)}\left[f_{\infty}^{-1}[y]\right]=\bigcup\left\{N_{\epsilon(y)}\left[f_{n}^{-1}[y]\right] \mid n=0,1, \cdots\right\} \subset f_{\infty}^{-1}[G], \quad \text { if } y \in G,
$$

and

$$
\begin{aligned}
d_{\infty}\left(f_{\infty}^{-1}[y], f_{\infty}^{-1}[z]\right) & =\inf \left\{d_{n}\left(f_{n}^{-1}[y], f_{n}^{-1}[z]\right) \mid n=0,1, \cdots\right\} \\
& \geqq \epsilon(y)-\epsilon(z), \quad \text { if } y, z \in G .
\end{aligned}
$$

Proposition 6. If $Y$ has a topology which makes $f$ a map (a quotient map), then $f_{\infty}$ is a map (a quotient map).

Proof. Trivial.

This completes the material needed to prove Theorem 3. In fact, we need from Proposition 5 only the fact that $X_{\infty}, d_{\infty}, Y, f_{\infty}$ satisfy part (i) of (C). Part (ii) is needed for $X, d, Y, f$ to maintain part (i) in the proof of Proposition 4. It is also worth noting that (C) is not needed in Propositions 3 and 6.

In [2] and [3] it is shown that, if $X$ is metrizable and $f$ is a closed map, then $Y$ is metrizable if $\mathrm{B} d f^{-1}[y]$ is compact for all $y \in Y$. This same result is true for pseudo-metrizable spaces if $Y$ is $T_{1}$ or regular. Hence, if $X$ is pseudo-metrizable, $Y$ is $T_{1}$ or regular, $f$ is a closed map, and each $\mathrm{B} d f^{-1}[y]$ is compact, it follows from the "only if" part of Theorem 3 that $X, d, Y, f$ satisfy (C) for some pseudo-metric $d$ compatible with $X$. It would be interesting to find directly a pseudometric $d$ such that $X, d, Y, f$ satisfy (C), and hence obtain a new proof of the result in [2] and [3]. It would be even more desirable to show (if such be the case) that $X, d, Y, f$ satisfy (C) for every pseudometric $d$ compatible with $X$. The best this author has been able to manage in this direction is the following.

TheоReм 4. Suppose that $X$ is pseudo-metrizable with pseudo-metric $d$, that $Y$ is a topological space, and that $f$ is continuous ( $f$ need not be closed). Then $X, d, Y, f$ satisfy (C) if there is a finite subset $A$ of $Y$ such that $f^{-1}[y]$ is compact for all $y \in A$ and diam $f^{-1}[y]=0$ for all $y \in Y-A$.

Proof. Let $A$ be as in the theorem, and let $G$ be an arbitrary open subset of $Y$. For each $y \in G$, let $\delta(y)>0$ be the largest real number such that $N_{\delta(y)}\left[f^{-1}[y]\right] \subset f^{-1}[G]$. Let $B=A \cap G$, and let $\epsilon>0$ be such that $\epsilon<\delta(y) / 2$ for all $y \in B$. Finally, define

$$
\begin{aligned}
\epsilon(y) & =\epsilon, \quad \text { if } f^{-1}[y] \subset N_{\mathrm{e}}\left[f^{-1}[B]\right], \\
& =\min \{\epsilon, \delta(y)\}, \quad \text { if } f^{-1}[y] \nsubseteq N_{\mathrm{e}}\left[f^{-1}[B]\right] .
\end{aligned}
$$


It is immediate that $\{\epsilon(y) \mid y \in G\}$ satisfies part (i) of (C). To check part (ii), let $y, z \in G$ and consider the following cases.

Case 1. $f^{-1}[y], f^{-1}[z] \subset N_{\epsilon}\left[f^{-1}[B]\right]$. Then

$$
\epsilon(y)-\epsilon(z)=0 \leqq d\left(f^{-1}[y], f^{-1}[z]\right) .
$$

Case 2. $f^{-1}[y] \nsubseteq N_{\epsilon}\left[f^{-1}[B]\right]$ and $f^{-1}[z] \subset N_{\epsilon}\left[f^{-1}[B]\right]$. Then $\operatorname{diam} f^{-1}[y]=0=\operatorname{diam} f^{-1}[z]$, and it follows easily that $\boldsymbol{\epsilon}(y)-\boldsymbol{\epsilon}(z)$ $\leqq d\left(f^{-1}[y], f^{-1}[z]\right)$.

Case 3. $f^{-1}[y] \subsetneq N_{\epsilon}\left[f^{-1}[B]\right], f^{-1}[z] \subset N_{\epsilon}\left[f^{-1}[B]\right]$. Hence $\operatorname{diam} f^{-1}[y]$ $=0$. Observe that $\epsilon-d\left(f^{-1}[y], f^{-1}[z]\right) \leqq \delta(y)$, since

$$
\begin{aligned}
d\left(u, f^{-1}[y]\right) & <\epsilon-d\left(f^{-1}[y], f^{-1}[z]\right) \Rightarrow d\left(u, f^{-1}[z]\right)<\epsilon \Rightarrow d\left(u, f^{-1}[B]\right) \\
& <2 \epsilon\left(\text { since } f^{-1}[z] \subset N_{\epsilon}\left[f^{-1}[B]\right]\right) \Rightarrow u \in f^{-1}[G] .
\end{aligned}
$$

Now, using the inequality $\epsilon-\delta(y) \leqq d\left(f^{-1}[y], f^{-1}[z]\right)$, it is easy to check that $\epsilon(z)-\epsilon(y) \leqq d\left(f^{-1}[z], f^{-1}[y]\right)$. The inequality $\boldsymbol{\epsilon}(y)-\epsilon(z)$ $\leqq d\left(f^{-1}[y], f^{-1}[z]\right)$ is trivial.

If $A$ is not finite in the preceding theorem, one apparently must use the hypothesis that $f$ is closed to prove $(\mathrm{C})$, since, in the example following Theorem 2 of [1], $f$ is open and continuous, $X$ is metrizable, and each $f^{-1}[y]$ is compact, but $Y$ is not normal and hence not pseudo-metrizable. In [3, p. 699] there is an example in which $f$ is open and continuous, $X$ is metrizable, each $f^{-1}[y]$ is compact, and $Y$ is Hausdorff but not normal.

On the other hand, the assumption that $f$ is a quotient map and that (C) is true implies neither that $f$ is closed nor that each $f^{-1}[y]$ is compact. Hence Theorem 3 is not contained in [2] and [3]. For an example, consider the usual projection of the plane on to the reals.

With regard to the question preceding Theorem 4 , it would be interesting to know, whether the truth of (C) for some $d$ implies (C) for all equivalent (or even uniformly equivalent) pseudo-metrics, under the more general hypothesis that $f$ is a quotient map rather than a closed map.

\section{REFERENCES}

1. C. J. Himmelberg, Quotient uniformities, Proc. Amer. Math. Soc. 17 (1966), 1385-1388.

2. K. Morita and S. Hanai, Closed mappings and metric spaces, Proc. Japan Acad. 32 (1956), 10-14.

3. A. H. Stone, Metrizability of decomposition spaces, Proc. Amer. Math. Soc. 7 (1956), 690-700.

UNiversity OF Kansas 